\title{
ON SPATIAL THINNING-REPLACEMENT PROCESSES BASED ON VORONOI CELLS
}

\author{
K. A. BOROVKOV, ${ }^{*}$ The University of Melbourne \\ D. A. ODELL, ${ }^{* *}$ MASCOS
}

\begin{abstract}
We introduce a new class of spatial-temporal point processes based on Voronoi tessellations. At each step of such a process, a point is chosen at random according to a distribution determined by the associated Voronoi cells. The point is then removed, and a new random point is added to the configuration. The dynamics are simple and intuitive and could be applied to modelling natural phenomena. We prove ergodicity of these processes under wide conditions.
\end{abstract}

Keywords: Point process; Voronoi tessellation; Markov chain; ergodicity

2000 Mathematics Subject Classification: Primary 60G55

Secondary 60J27; 60F99

\section{Introduction}

A spatial point process is a stochastic model for the location of events in a space, and as such it is a random element which takes discrete sets of points as its values. Trees in a forest, schools in a city, or capillaries in a cross-section of a bodily organ are examples of situations that can be modelled by a point process over a two-dimensional manifold; impurities in metals or positions of submarines require models defined over three-dimensional manifolds. The one-dimensional point process is often used as a model for events in time, but may also be applied to such problems as the physical distribution of files on a hard-disk, where the underlying space can be treated as an interval in $\mathbb{R}$.

The Voronoi tessellation is a useful tool for the analysis of spatial point processes. This can be defined for any locally finite set of points $X$ in a metric space $(M, d)$. With each $x \in X$ we associate its Voronoi cell

$$
C_{x}^{X}(M):=\left\{y \in M: d(y, x)=\min \left[d\left(y, x^{\prime}\right): x^{\prime} \in X\right]\right\} .
$$

(We will generally suppress the reference to $M$ in the notation for the cell and just write $C_{x}^{X}$ where the underlying set is clear from the context.) The tessellation produced by $X$ is $T_{X}=\left\{C_{x}^{X}: x \in X\right\}$, and the elements of $X$ are called the generators of $T_{X}$. Statistics drawn from analysis of this structure provide an intrinsic description of the distribution pattern of the set of generators and have accordingly been investigated extensively (see e.g. [8], [10], [11], [12], [13]). The Voronoi tessellation is also a natural object of interest whenever concepts such as catchment area or zone of influence are appropriate to the situation being modelled, as in most of the examples mentioned above. When this is the case it is also very interesting to consider

Received 9 June 2006; revision received 15 December 2006.

* Postal address: Department of Mathematics and Statistics, The University of Melbourne, Parkville, VIC 3010, Australia. Email address: kostya@ms.unimelb.edu.au

** Postal address: MASCOS, 139 Barry Street, Carlton, VIC 3010, Australia. 
point processes evolving in time under laws which are determined by characteristics of the Voronoi tessellation. A simple example is a process in which the lifetime of a point is a random variable with distribution determined by its Voronoi cell. Examples of this kind of spatialtemporal point process are the Hotelling processes [6], [9], which model the geographical distribution of businesses competing for market share and the adjustment models for territorial animal behaviour [5] (for further examples, see e.g. [10], [14]). One could also mention here another family of dynamic point processes whose dynamics is related to the respective Voronoi tessellations, and which converge to the so-called centroidal Voronoi diagrams (where the generators are at the same time centroids of their cells). These include the random $k$-means algorithm and stochastic versions of Lloyd's algorithm; see e.g. [4].

As far as we know there has been no general treatment of Voronoi tessellation-based dynamic models, although they promise to produce a rich class of stochastic objects typifying various kinds of spatial clustering.

In this paper we consider some classes of such models viewed as discrete-time Markov processes $\left\{\boldsymbol{X}_{n}\right\}_{n \geq 0}$, taking values in a fixed finite-dimensional configuration space. Let $(M, d)$ be a metric space and $N$ a fixed positive integer representing the initial number of points in $M$. The associated configuration space will be either $X=\bigcup_{k=1}^{N} M^{k}$ or $X=M^{N}$, depending on whether the process is of a thinning or thinning-replacement type. Any finite set of points $X$ generating a Voronoi tessellation can of course be represented in many different ways as a point $\boldsymbol{x} \in X$, since the order of the points is immaterial to the tessellation; however, it is convenient to retain an ordering of the points in the configuration space. In the thinning-replacement process the total number of points after each replacement remains constant, although the thinning and replacement components are independent stochastic processes. Replacement will always be determined by a probability measure $\mu$ on $M$, equivalent to the volume measure, whereas the thinning follows a probability rule on the Voronoi tessellation. We tacitly assume that all subsets of $M$ or $X$ appearing in this paper are measurable.

Let a selection function $S: C \rightarrow \mathbb{R}^{+}$be given, defined on the space $C$ of all possible Voronoi cells from tessellations generated by finite sets in $M$. If $\boldsymbol{x}=\left(x_{1}, \ldots, x_{k}\right)$ is the current configuration, then in the next step exactly one point $x_{J}, 1 \leq J \leq k$, is chosen at random and either removed (in a thinning process) or reassigned (in a thinning-replacement process) to a random position. The probability of choosing the generator $x_{j}$ is proportional to the value of the selection function $S\left(C_{x_{j}}^{x}\right)$ on the respective Voronoi cell $C_{x_{j}}^{x}$, i.e.

$$
\operatorname{Pr}(J=j \mid \boldsymbol{x}):=\frac{S\left(C_{x_{j}}^{\boldsymbol{x}}\right)}{\sum_{i=1}^{k} S\left(C_{x_{i}}^{\boldsymbol{x}}\right)} .
$$

There are many quantifiable properties of a Voronoi cell upon which the function $S$ can be based. Some of these, such as volume, perimeter or surface area or suitable higher dimensional generalisation, number of edges, number of faces, minimal or maximal internal angles etc., are properties of any closed simplicial complex in the same underlying space. Others, such as the volume of the associated Voronoi flower (the Voronoi flower of $C_{x_{j}}^{\boldsymbol{x}}$ is the closure of the set $\left\{z \in M: C_{x_{j}}^{x \cup\{z\}} \neq C_{x_{j}}^{x}\right\}$ ) or the distance to nearest neighbour, are specific to the Voronoi structure but can be computed from the properties of the individual cell. A further generalisation could allow $S$ to be a function of $x_{j}$ and of $\boldsymbol{x}$, for example the weighted sum of cell functions in the original sense with weights determined by the number of edges in the smallest nearestneighbour arc to $x_{j}$. In this sense, the degree of a selection function $S$ could be defined as the depth of the Voronoi nearest-neighbour relation required to fix its value. We will consider 
only the simplest kinds of first-degree selection functions. More specifically, we consider the following two classes of such processes.

(A) The 'volume-based', or $v$-process class. We require enough additional structure for $(M, d)$ so that cell volume can be defined. Let $M$ be a compact, piecewise-smooth manifold equipped with a measure $\lambda$, which is equivalent to the volume measure on $M$ with a density bounded away from zero and from infinity. We assume that the value of $S\left(C_{x_{j}}^{x}\right)$ is determined by the value of $\lambda\left(C_{x_{j}}^{x}\right)$ :

$$
S\left(C_{x_{j}}^{x}\right)=S_{v}\left(\lambda\left(C_{x_{j}}^{x}\right)\right) .
$$

Without ambiguity we drop the subscript in $S_{v}$ and consider $S: \mathbb{R}^{+} \rightarrow \mathbb{R}^{+}$to be a function of $\lambda\left(C_{x_{j}}^{x}\right)$. If $S$ is increasing, then points with Voronoi cells of large volume are more likely to be chosen to be culled or moved, and so the selection pressure favours points with small cells, that is, points restricted by close neighbours. A decreasing $S$ favours points with large cells, that is, isolated points or points whose near neighbours fall within a limited arc. Functions of the form $S(v)=v^{\alpha}, \alpha \in \mathbb{R}$, produce scale-independent models.

(B) The neighbour-based, or $n$-process class. The Voronoi tessellation determines for each generator a set of its Voronoi-nearest neighbours. Formally, this set is defined as

$$
\left[x_{j}\right]^{x}:=\left\{x_{i} \text { from } x \text { with } i \neq j \text {, such that } C_{x_{i}}^{x} \text { and } C_{x_{j}}^{x} \text { have a common facet }\right\} .
$$

We now assume that $S\left(C_{x_{j}}^{x}\right)=S_{n}\left(\operatorname{card}\left(\left[x_{j}\right]^{x}\right)\right)$, for some $S_{n}:\{1, \ldots, N\} \rightarrow \mathbb{R}^{+}$, where again we drop the subscript when the context is clear. The $n$-process requires less structure on $(M, d)$, but the selection function $S$ determines which, if any, types of cells are favoured by the evolution.

Figure 1 to Figure 4 below illustrate some of the behaviours that were observed in simulations. Figure 1 depicts side-by-side realisations of three different $v$-processes on a circle with $S(v)=$ $v^{\alpha}$, each having the same total number of points $(N=128)$, but different $\alpha$ values. The base of each rectangle represents the circle $M$ opened out into a line segment by a cut, whereas the $y$-axis represents the time. The well-defined clustering observed in (c) was found to occur when $\alpha>1$ and $N$ is sufficiently large (for example, for $\alpha=1.5, N=10$ ). The phase change was observable even for values of $\alpha$ close to one, as seen in Figure 2, where the time scale has been compressed by a factor of five from that of Figure 1, to bring it out more clearly.

Figure 3 shows the results of running four instances of the $v$-process on the unit square with $S(v)=v^{\alpha}$ and values of $\alpha$ ranging from -3.0 to 1.4 . The phase change at $\alpha=1$ was observed just as in the one-dimensional case. For values of $\alpha \leq 1$, a smooth gradation of the degree of clustering was observed without apparent interference from edge effects.

Two instances of the $n$-process are shown in Figure 4. The $n$-processes produced a rich collection of different clustering behaviours, among which it appears that selections which favour cells with a greater than average number of neighbours (the average being six) led to less clustered configurations, and vice versa.

In [3] we discuss the simulation results in more detail, and consider the problem of inferring the selection function from the statistics of a given point pattern which has arisen from a $v$ - or $n$-process elsewhere. In particular, we employ Thiel's redundancy measure [6], [10] and Baddeley and van Lieshout's $J$-function [2] as a first step in the characterisation of these patterns.

Simulations appear to demonstrate ergodic-type behaviour for $v$-processes with $S(v)=v^{\alpha}$ when $\alpha \leq 1$, whereas for $\alpha>1$ the behaviour has a very different character which indicates 


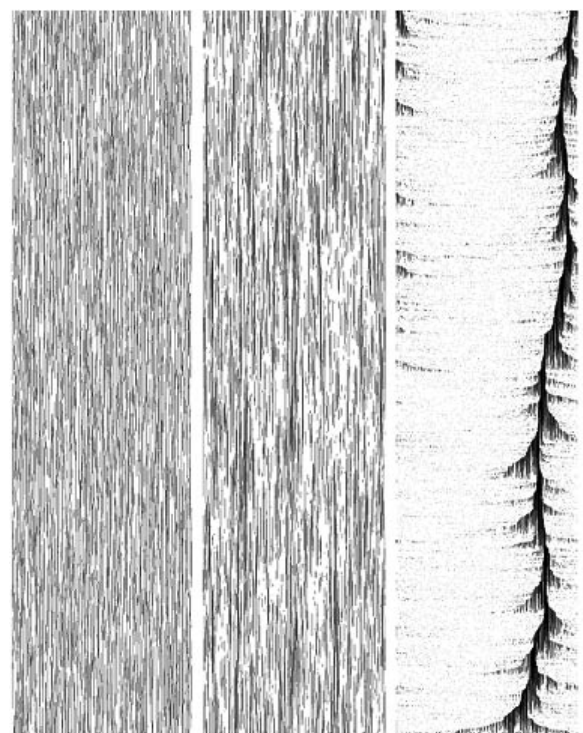

(a)

(b)

(c)

FIGURE 1: Evolution of $v$-processes on a circle. Each of the three processes (for the selection functions $S(v)=v^{\alpha}$ with (a) $\alpha=-1.0$, (b) $\alpha=0.5$, (c) $\alpha=1.5$, respectively) was run with $N=128$ points for $T=4096$ steps (the vertical axis represents time).

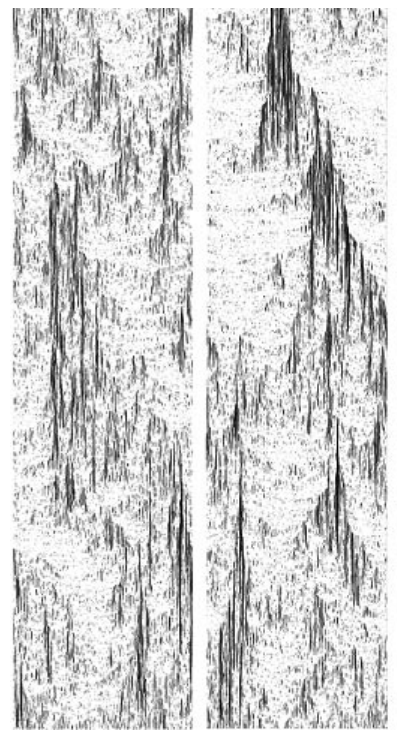

(a) (b)

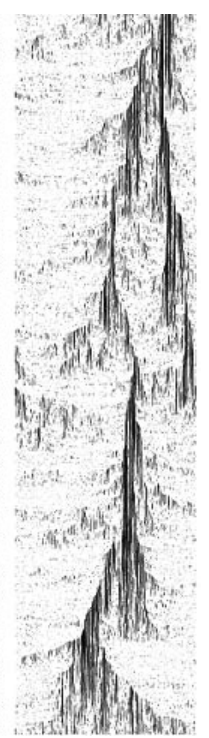

(c)

Figure 2: Phase change in the $v$-process on circle. Here $N=128, T=20480$, and $S(v)=v^{\alpha}$ with (a) $\alpha=0.95$, (b) $\alpha=1.0$, (c) $\alpha=1.05$. 


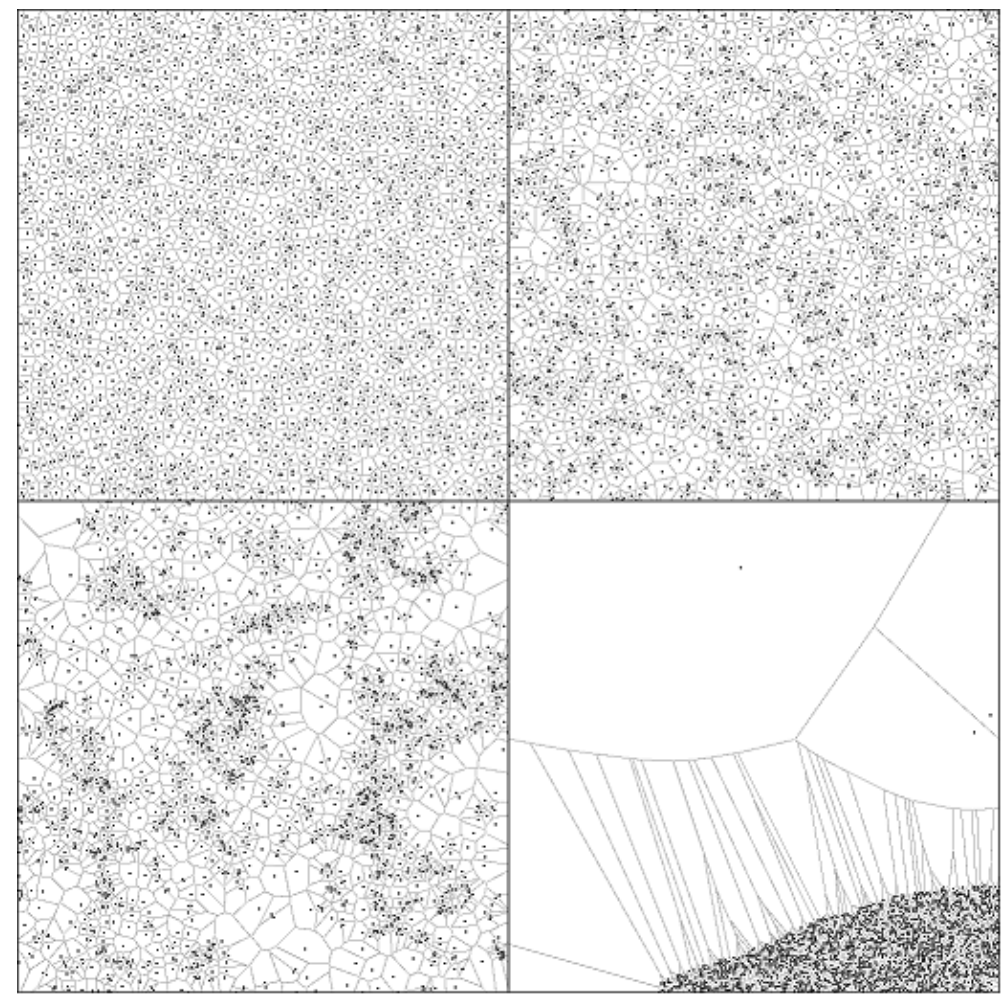

FIGURE 3: Realisations of four $v$-processes on $[0,1]^{2}$ with $N=2000, T=24000$, and $S(v)=v^{\alpha}$ for $\alpha=-3.0,0.2,1.0,1.4$ (from left to right and top to bottom).

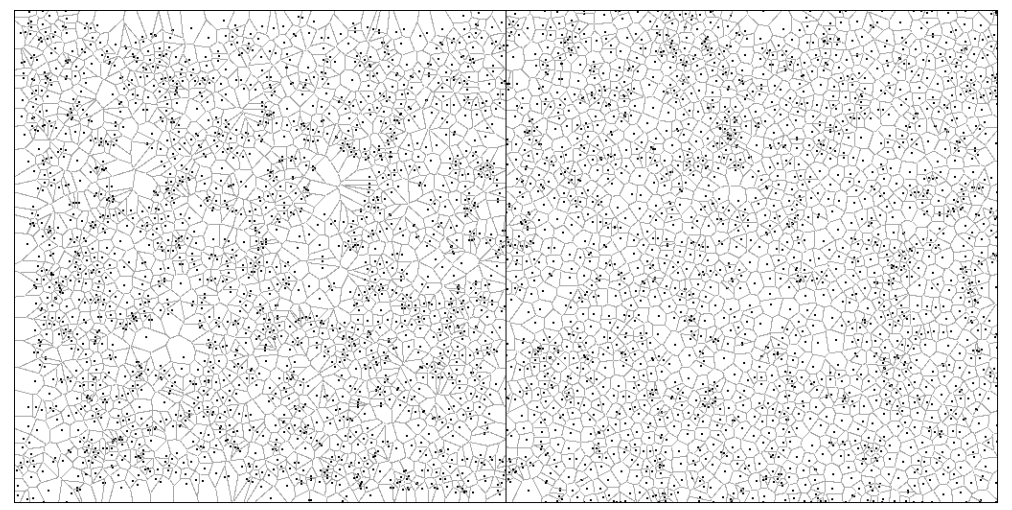

FIGURE 4: Realisations of two $n$-processes with $N=2000$ and $T=16000$. The pattern on the left was produced using the selection function $S(n)=0.1+|n-6|^{2}$ which 'favours' cells with six or close to six sides, whilst that on the right was produced by $S(n)=1$, for $n \neq 5$ with $S(5)=5000$, which strongly 'disfavours' cells with five sides. Note the apparent edge-effects in the former case. 
that ergodicity may not take place. In the present paper we solve the problem of ergodicity raised by these observations.

In Section 2 we prove, under rather broad conditions, the ergodicity for both the $n$ - and $v$-processes. In Section 3 we present a crude explanatory model which explains the phase change observed at $\alpha=1$ in the $v$-process with $S(v)=v^{\alpha}$.

Remark 1. Our process with replacement resembles a Gibbs sampler in that at each step only one coordinate of the state is resampled. The main difference is that in a Gibbs sampler the choice of the coordinate to be resampled is deterministic, but the new sample is from a distribution conditioned on the other current points. In our case, the choice of point is from a distribution conditioned by the set of current points, and the new sample is independent, identically distributed. This latter choice could easily be modified so that the new positions of the resampled coordinates are governed by a general set of conditional distributions, as in the usual application of the Gibbs sampler.

\section{Main results}

In this section we consider only processes with replacement. Assume that $M$ is a compact, piecewise- $C^{2}$ manifold in $\mathbb{R}^{m}, m \geq 1$, with or without boundary, endowed with the geodesic metric $d$. Let $\mu$ be a probability and $\lambda$ a measure on $M$, both equivalent to the volume measure $v$ on the manifold.

We define a discrete-time Markov $n$ - or $v$-process with state space $X=M^{N}$ as follows. Let $\boldsymbol{x} \in X, A$ be a Borel subset of $X, z \in M$, and $\Delta \subseteq M$, such that

$$
\begin{aligned}
x[j, z] & :=\left(x_{1}, \ldots, x_{j-1}, z, x_{j+1}, \ldots, x_{N}\right), \\
A_{j}[x] & :=\{z \in M: x[j, z] \in A\} \quad \text { and } \\
A[j, \Delta]: & =\{\boldsymbol{x}[j, z] \in X: \boldsymbol{x} \in A, z \in \Delta\} .
\end{aligned}
$$

We also write, with a slight abuse of notation, $x \in \boldsymbol{x}$ when $\boldsymbol{x}=\left(x_{1}, \ldots, x_{N}\right)$ and $x=x_{j}$ for some $j, 1 \leq j \leq N$.

The transition probability function is given by

$$
P(\boldsymbol{x}, A):=\operatorname{Pr}\left(\boldsymbol{X}_{n+1} \in A \mid \boldsymbol{X}_{n}=\boldsymbol{x}\right)=\sum_{j=1}^{N} \mu\left(A_{j}[\boldsymbol{x}]\right) \operatorname{Pr}(J=j \mid \boldsymbol{x}),
$$

where $\operatorname{Pr}(J=j \mid \boldsymbol{x})$ is the selection probability function (1).

Theorem 1. (i) The n-process with replacement with an arbitrary selection function

$$
S:\{1, \ldots, N\} \rightarrow \mathbb{R}^{+}
$$

which takes only positive values is Harris ergodic.

(ii) The v-process with replacement with a selection function $S:(0, \lambda(M)] \rightarrow \mathbb{R}^{+}$, such that both $S$ and $1 / S$ are locally bounded, is Harris ergodic.

Recall that Harris ergodicity entails convergence to the stationary distribution in total variation (see, e.g. p. 560 of [7] or p. 154 of [1]).

Remark 2. It is not difficult to see that the assertion of part (i) holds under more general conditions as well. 
Proof. (i) Denote by $\mu^{\otimes N}$ the $N$-fold product measure on $X$, and by $P^{(k)}$, the $k$-step transition function generated by $P$. We start with the following lemma.

Lemma 1. The n-process with replacement, with $M$ and $\mu$ as specified above, satisfies the following Döblin condition: there exist $\epsilon<1$ and $\gamma>0$ such that if $\mu^{\otimes N}(A)>\epsilon$ for a measurable $A \subseteq X$, then $P^{(N)}(\boldsymbol{x}, A) \geq \gamma$ for any $\boldsymbol{x} \in X$.

Proof. Let $B(z, \delta)=\{x \in M: d(x, z)<\delta\}$ be the $\delta$-ball around the point $z \in M$, and let $H=\prod_{i=1}^{N} B\left(z_{i}, \delta_{i}\right) \subset X$, for $z_{i} \in M$ and $\delta_{i}>0$. It suffices to find a constant $k_{0}>0$, independent of $\left\{z_{i}, \delta_{i}: 1 \leq i \leq N\right\}$, such that, for any $\boldsymbol{x} \in X$,

$$
P^{(N)}(\boldsymbol{x}, H)>k_{0} \mu^{\otimes N}(H) .
$$

Indeed, since any open subset of $X$ can be approximated in $\mu^{\otimes N}$-measure arbitrarily well by disjoint unions of basic sets $H$, by the regularity of the measures on $X$ (3) implies that

$$
P^{(N)}(\boldsymbol{x}, A)>k_{0} \mu^{\otimes N}(A),
$$

for any $\boldsymbol{x} \in X$ and $A \subseteq X$.

First, note that for any $\boldsymbol{x}=\left(x_{1}, x_{2}, \ldots, x_{N}\right) \in X$ and $\Delta \subseteq M$, by (1) and (2),

$$
P(\boldsymbol{x},\{\boldsymbol{x}\}[j, \Delta]) \geq k \mu(\Delta),
$$

where

$$
k=\frac{\min _{j \leq N} S(j)}{N \max _{j \leq N} S(j)}>0 .
$$

Therefore, for any $y \in M$ and $i \neq j$ we also have $P(\boldsymbol{x}[i, y],\{\boldsymbol{x}[i, y]\}[j, \Delta]) \geq k \mu(\Delta)$. Considering that the Markov chain can proceed from $\boldsymbol{x}$ to the set $\{\boldsymbol{x}\}\left[i, B\left(z_{i}, \delta_{i}\right)\right]\left[j, B\left(z_{j}, \delta_{j}\right)\right]$ by first changing the value of the $i$ th component and then that of the $j$ th one, or vice versa, from (5) we obtain

$$
\begin{aligned}
P^{(2)}(\boldsymbol{x}, & \left.\{\boldsymbol{x}\}\left[i, B\left(z_{i}, \delta_{i}\right)\right]\left[j, B\left(z_{j}, \delta_{j}\right)\right]\right) \\
= & \int_{B\left(z_{i}, \delta_{i}\right)} \int_{B\left(z_{j}, \delta_{j}\right)} P\left(\boldsymbol{x},\{\boldsymbol{x}\}\left[j, \mathrm{~d} y_{j}\right]\right) P\left(\boldsymbol{x}\left[j, y_{j}\right],\{\boldsymbol{x}\}\left[j, y_{j}\right]\left[i, \mathrm{~d} y_{i}\right]\right) \\
& +\int_{B\left(z_{j}, \delta_{j}\right)} \int_{B\left(z_{i}, \delta_{i}\right)} P\left(\boldsymbol{x},\{\boldsymbol{x}\}\left[i, \mathrm{~d} y_{i}\right]\right) P\left(\boldsymbol{x}\left[i, y_{i}\right],\{\boldsymbol{x}\}\left[i, y_{i}\right]\left[j, \mathrm{~d} y_{j}\right]\right) \\
\geq & 2 k^{2} \int_{B\left(z_{i}, \delta_{i}\right)} \mu\left(\mathrm{d} y_{i}\right) \int_{B\left(z_{j}, \delta_{j}\right)} \mu\left(\mathrm{d} y_{j}\right) \\
= & 2 k^{2} \mu\left(B\left(z_{i}, \delta_{i}\right)\right) \mu\left(B\left(z_{j}, \delta_{j}\right)\right) .
\end{aligned}
$$

Continuing in this way, we will clearly get

$$
P^{(N)}\left(\boldsymbol{x}, \prod_{j=1}^{N} B\left(z_{j}, \delta_{j}\right)\right) \geq N ! k^{N} \prod_{j=1}^{N} \mu\left(B\left(z_{j}, \delta_{j}\right)\right),
$$

which proves (3) and the lemma. 
Relation (4) clearly implies the irreducibilty of the $n$-process. Indeed, (4) also implies that, for any $\boldsymbol{x} \in X$ and $A \subseteq X$,

$$
P^{(N+1)}(\boldsymbol{x}, A)>k \mu^{\otimes N}(A),
$$

and hence the process is aperiodic. Now the desired assertion is immediate from Theorem 16.0.2 of [7, p. 391]. Part (i) of Theorem 1 is proved.

(ii) Ergodicity of the $v$-process requires more analysis. We wish to produce an argument similar to that of Lemma 1 . The measure $\mu$ determines probabilities for points entering the configuration (the replacement points) and it will be sufficient for our arguments to note that, for any fixed $\delta$,

$$
\mu_{\delta}:=\inf \{\mu(B(x, \delta)): x \in M\}>0,
$$

as a consequence of the smoothness and compactness of $M$. Similarly we can define

$$
\lambda_{\delta}:=\inf \{\lambda(B(x, \delta)): x \in M\}>0 .
$$

For the $v$-process we do not have a simple analogy to the lower bound derived from (6). Here what is required is a positive lower bound for $\lambda\left(B(x, \delta) \cap C_{x}^{\boldsymbol{x}}\right)$, which does not exist if $\boldsymbol{x}$ is unrestricted. For example, if $x \in \partial M$, then a single other point in the configuration $x$ can cause the Voronoi cell around $x$ to be arbitrarily small in volume and, consequently, the value of the selection function may become arbitrarily small on this cell. So first we need to define those points which are $\delta$-far from the boundary.

For $\delta>0$, let $M_{\delta}:=\{x \in M: d(x, y)>\delta$ for any $y \in \partial M\}$ be the inner parallel set to $M$; if $M$ is without a boundary, then $M_{\delta}:=M$. The smoothness of $M$ guarantees that $\mu\left(M_{\delta}\right)>0$ and $\lambda\left(M_{\delta}\right)>0$ for $\delta$ small enough. Take any $x \in M_{\delta}$ and $x \in X$, and define

$$
n(x, \boldsymbol{x}, \delta):= \begin{cases}\operatorname{card}(\{y \in \boldsymbol{x}: 0<d(x, y)<2 \delta\}) & \text { for } x \in \boldsymbol{x} \\ N & \text { otherwise }\end{cases}
$$

So if $\boldsymbol{x}$ is such that $n(x, \boldsymbol{x}, \delta) \leq 1$, then $B(x, \delta)$ intersects with at most two of the Voronoi cells $C_{x_{j}}^{x}$. This means that if $x \in M_{\delta}$ is fixed, then for all $\delta>0$ sufficiently small, and any $\boldsymbol{x} \in X$ such that $n(x, \boldsymbol{x}, \delta) \leq 1$, we have, via the smoothness of $M$,

$$
\lambda\left(B(x, \delta) \cap C_{x}^{\boldsymbol{x}}\right) \geq \lambda_{\delta / 3} .
$$

For $\delta>0$, define

$$
\mathcal{D}_{\delta}:=\left\{\left(x_{1}, \ldots, x_{N}\right) \in X: \text { all } x_{i} \in M_{\delta} \text { and } d\left(x_{i}, x_{j}\right)>2 \delta, i \neq j\right\} .
$$

Clearly we can choose $\delta$ small enough that $\mu^{\otimes N}\left(\mathcal{D}_{\delta}\right)>0$. Now define a new measure $\phi$ on $X$ as follows:

$$
\phi(A):=\mu^{\otimes N}\left(A \cap \mathcal{D}_{\delta}\right) .
$$

Lemma 2. (i) There are $\delta>0$ and $\epsilon>0$ such that, for every $x \in X$,

$$
P^{(N)}\left(\boldsymbol{x}, \mathcal{D}_{\delta}\right)>\epsilon .
$$

(ii) There exists a $\gamma>0$ such that for $\delta$ as above and any $\boldsymbol{x} \in \mathcal{D}_{\delta}$, and any Borel subset $A \subseteq X$,

$$
P^{(N)}(\boldsymbol{x}, A) \geq \gamma \phi(A) .
$$


Proof. (i) Let $\delta_{0}>0$ be small enough that $M_{\delta_{0}}$ has positive $\mu$ measure (and hence positive $\lambda$ measure). By appealing to the smoothness structure of $M$ or otherwise we can find $N+1$ disjoint open subsets of $M_{\delta_{0}}$. Denote these by $L_{1}, L_{2}, \ldots, L_{N+1}$ and fix them for the rest of the proof. Let

$$
\delta_{i}:=\sup \left\{\delta: \text { there are } N \text { disjoint balls } B\left(z_{k}, 3 \delta\right) \subset L_{i}\right\}, \quad i=1, \ldots, N+1 .
$$

Clearly,

$$
\delta:=\min _{0 \leq i \leq N+1} \delta_{i}>0 .
$$

Now, for any $\boldsymbol{x}=\left(x_{1}, \ldots, x_{N}\right) \in X$, at least one of the $L_{i}$ will be disjoint from $\left\{x_{1}, \ldots, x_{N}\right\}$. Therefore, for such an $i$, we can find $z_{1}^{(i)}, \ldots, z_{N}^{(i)} \in L_{i}$ such that

$$
B\left(z_{j}^{(i)}, 3 \delta\right) \cap B\left(z_{k}^{(i)}, 3 \delta\right)=\varnothing \quad \text { for } j \neq k,
$$

and

$$
x_{k} \notin B\left(z_{j}^{(i)}, 3 \delta\right) \text { for all } k, j \in\{1, \ldots, N\} .
$$

Obviously, $\prod_{j=1}^{N} B\left(z_{j}^{(i)}, \delta\right) \subset \mathcal{D}_{\delta}$.

As in Lemma 1, we consider a possible sequence of $N$ steps of the Markov process in each of which exactly one of the 'coordinate points' $x_{m}$ of $\boldsymbol{x}$ moves into a unique $B\left(z_{j^{\prime}}^{(i)}, \delta\right)$, $j=1, \ldots, N$. At each step, the probability of the chosen point landing in a $B\left(z_{j^{\prime}}^{(i)}, \delta\right)$ is greater than or equal to $\mu_{\delta}$, so we need only be concerned with the probability of all $N$ original points moving in $N$ consecutive steps of the process. This will have a lower bound independent of the initial state $\boldsymbol{x}$ if we can find a bound $\kappa>0$ with the following property: whenever we are at a stage at which some, but not all, of the $x_{m}$ have moved and are in corresponding sets $B\left(z_{j^{\prime}}^{(i)}, \delta\right)$, there exists a $j$ such that $x_{j}$ has not moved yet, and

$$
\operatorname{Pr}\left(J=j \mid \boldsymbol{x}^{\prime}\right)>\kappa, \quad \text { where } \boldsymbol{x}^{\prime} \text { is the current state of the process. }
$$

Let us assume that in the first $k \geq 1$ steps, $k$ different original points $x_{i_{1}}, \ldots, x_{i_{k}}$ have moved. We denote their new positions by $x_{i_{1}}^{\prime}, \ldots, x_{i_{k}}^{\prime}$. Write $v:=\left\{i_{1}, \ldots, i_{k}\right\}, \xi:=\{1, \ldots, N\} \backslash v=$ $\left\{j: x_{j}\right.$ has not moved $\}$, and

$$
S_{\Sigma}:=\sum_{j=1}^{N} S\left(\lambda\left(C_{x_{j}}^{x^{\prime}}\right)\right)=\left(\sum_{j \in v}+\sum_{j \in \xi}\right) S\left(\lambda\left(C \frac{x^{\prime}}{x_{j}}\right)\right)=: S_{\Sigma}^{\nu}+S_{\Sigma}^{\xi} .
$$

Then $p:=S_{\Sigma}^{\xi} / S_{\Sigma}=\operatorname{Pr}\left(J \in \xi \mid \boldsymbol{x}^{\prime}\right)$ is the probability that at the next step one of the remaining points will move.

If $p>\frac{1}{2}$, then, for some $j \in \xi$,

$$
\operatorname{Pr}\left(J=j \mid \boldsymbol{x}^{\prime}\right) \geq \frac{1}{2 \operatorname{card}(\xi)} \geq \frac{1}{2 N} ;
$$

otherwise

$$
S_{\Sigma}^{\xi} \leq S_{\Sigma}^{\nu}
$$

We have only to consider the latter case. For an $x_{j}^{\prime}, j \in v$, we have

$$
x_{j}^{\prime} \in B\left(z_{j^{\prime}}^{(i)}, \delta\right)
$$


for some $j^{\prime}$, and so not only do we have

$$
d\left(x_{j}^{\prime}, x_{m}^{\prime}\right)>2 \delta,
$$

when $j \neq m \in v$, but also for $m \in \xi$, since $B\left(z_{j^{\prime}}^{(i)}, 3 \delta\right)$ was free of any $x_{k}$ from the original state $\boldsymbol{x}$. This implies that for the set $W:=\bigcup_{j \in \xi} B\left(x_{j}, \delta\right)$ we have $C_{x_{i}^{\prime}}^{x^{\prime}} \cap W=\varnothing$ for $i \in v$, and therefore

$$
W \subset \bigcup_{j \in \xi} C_{x_{j}}^{x^{\prime}}
$$

Hence,

$$
\max _{j \in \xi} \lambda\left(C_{x_{j}}^{\boldsymbol{x}^{\prime}}\right) \geq \frac{\lambda(W)}{\operatorname{card}(\xi)} \geq \frac{\lambda_{\delta}}{\operatorname{card}(\xi)} \geq \frac{\lambda_{\delta}}{N},
$$

while, for all $j \in v$,

$$
\lambda\left(C_{x_{j}^{\prime}}^{x^{\prime}}\right) \geq \lambda_{\delta}
$$

Owing to the local boundedness of $S$ and $S^{-1}$,

$$
0<b:=\inf _{\lambda_{\delta} / N \leq v \leq \lambda(M)} S(v) \leq \sup _{\lambda_{\delta} / N \leq v \leq \lambda(M)} S(v)=: B<\infty .
$$

Therefore, by (1) and (11),

$$
\max _{j \in \xi} \operatorname{Pr}\left(J=j \mid \boldsymbol{x}^{\prime}\right) \geq \frac{b}{S_{\Sigma}^{\xi}+\operatorname{card}(v) B} \geq \frac{b}{2 N B}=: \kappa>0 .
$$

Clearly $\kappa \leq 1 / 2 N$, so from (10) and (12) we have, by a similar argument to that in the proof of Lemma 1 ,

$$
P^{(N)}\left(\boldsymbol{x}, \mathcal{D}_{\delta}\right) \geq N ! \kappa^{N-1} \mu_{\delta}^{N},
$$

which proves (8).

(ii) Let $A \subset X$ and assume that $\phi(A)>0$. As in the proof of Lemma 1, it will suffice to restrict attention to basic open sets

$$
H:=\prod_{i=1}^{N} B\left(w_{i}, r_{i}\right) \subset \mathcal{D}_{\delta}, \quad w_{i} \in M_{\delta} .
$$

As in the proof of (i), we specify a possible sequence of $N$ moves in the Markov process and aim to produce a bound analogous to (13) for $P^{(N)}(\boldsymbol{x}, H)$. The term $\mu_{\delta}^{N}$ in (13) is easily seen to be replaceable in this case by

$$
\prod_{i=1}^{N} \mu\left(B\left(w_{i}, r_{i}\right)\right)=\phi(H)
$$

since it represents the product of the probabilities that the points move into the target sets; the value $\kappa$ from (i) is no longer valid, however, because, retaining the notation above, we do not necessarily have $d\left(x_{j}, x_{m}^{\prime}\right)>2 \delta$ when $j \in \xi$ and $m \in v$. Thus, if we can find a new constant $\kappa^{\prime}>0$ such that, at every intermediate state $\boldsymbol{x}^{\prime}$,

$$
\max _{j \in \xi} \operatorname{Pr}\left(J=j \mid \boldsymbol{x}^{\prime}\right)>\kappa^{\prime}
$$


we will have

$$
P^{(N)}(\boldsymbol{x}, H)>N ! \kappa^{\prime N-1} \phi(H),
$$

and (9) will have been proven.

As before, let $\boldsymbol{x}^{\prime}$ be the state of the process after $k$ steps, and let $j \in \xi$. Since $\boldsymbol{x} \in \mathcal{D}_{\delta}$, for any other $i \in \xi$ we have $d\left(x_{j}, x_{i}\right)>2 \delta$. On the other hand, for all $l, m \in v$ we also have $d\left(x_{l}^{\prime}, x_{m}^{\prime}\right)>2 \delta$, as these points will be part of the configuration that we will get after $N$ steps, which has to belong to $H \subset \mathcal{D}_{\delta}$. Let $m \in v$ be such that

$$
d\left(x_{m}^{\prime}, x_{j}\right)=\min _{i \in v} d\left(x_{i}^{\prime}, x_{j}\right) .
$$

If $d\left(x_{m}^{\prime}, x_{j}\right)<\delta / 2$ then, for all other $i \in v, d\left(x_{i}^{\prime}, x_{j}\right)>3 \delta / 2$, and so the ball $B\left(x_{j}, 3 \delta / 4\right)$ is contained entirely within $C_{x_{j}}^{\boldsymbol{x}^{\prime}} \cup C_{x_{m}^{\prime}}^{\boldsymbol{x}^{\prime}}$, and hence, for sufficiently small $\delta>0$, by (7),

$$
\lambda\left(C_{x_{j}}^{\boldsymbol{x}^{\prime}}\right) \geq \lambda_{\delta / 8}
$$

Alternatively, $d\left(x_{i}^{\prime}, x_{j}\right) \geq \delta / 2$ for all $i \in v$, and so $B\left(x_{j}, \delta / 4\right) \subseteq C_{x_{j}}^{\boldsymbol{x}^{\prime}}$. In any case, $\lambda\left(C_{x_{j}}^{\boldsymbol{x}^{\prime}}\right) \geq$ $\lambda_{\delta / 8}$, and hence

$$
\operatorname{Pr}\left(J=j \mid \boldsymbol{x}^{\prime}\right) \geq \frac{\lambda_{\delta / 8}}{S_{\Sigma}}
$$

We also have

$$
\begin{aligned}
S_{\Sigma} & \leq \operatorname{card}(\xi) \sup _{v \in\left[\lambda_{\delta / 8}, \lambda(M)\right]} S(v)+\operatorname{card}(v) \sup _{v \in\left[\lambda_{\delta}, \lambda(M)\right]} S(v) \\
& \leq N \sup _{v \in\left[\lambda_{\delta / 8}, \lambda(M)\right]} S(v)=: Q<\infty .
\end{aligned}
$$

So we can take $\kappa^{\prime}:=Q^{-1} \inf _{v \in\left[\lambda_{\delta / 8}, \lambda(M)\right]} S(v)$ in (14). Lemma 2 is proved.

Following Section 5.4.3 of [7], aperiodicity for the $v$-process is defined as follows. Let

$$
\begin{array}{r}
E_{\mathcal{D}_{\delta}}:=\left\{n \geq 1: \text { there is a } \gamma_{n}>0 \text { such that } P^{(n)}(\boldsymbol{x}, A) \geq \gamma_{n} \phi(A)\right. \\
\text { for any } \left.\boldsymbol{x} \in \mathcal{D}_{\delta} \text { and } A \subset X\right\} .
\end{array}
$$

The $v$-process is called aperiodic if g.c.d. $\left(E_{\mathcal{D}_{\delta}}\right)=1$.

Lemma 3. The v-process is aperiodic.

Proof. It is enough to find $\gamma_{N+1}>0$ such that for any $\boldsymbol{x} \in \mathcal{D}_{\delta}$, and any Borel subset $A \subseteq X$,

$$
P^{(N+1)}(\boldsymbol{x}, A) \geq \gamma_{N+1} \phi(A) .
$$

From Lemma 2(ii),

$$
\begin{aligned}
P^{(N+1)}(\boldsymbol{x}, A) & =\int_{X} P(\boldsymbol{x}, \mathrm{d} \boldsymbol{y}) P^{(N)}(\boldsymbol{y}, A) \\
& \geq \int_{\mathcal{D}_{\delta}} P(\boldsymbol{x}, \mathrm{d} \boldsymbol{y}) P^{(N)}(\boldsymbol{y}, A) \\
& \geq P\left(\boldsymbol{x}, \mathcal{D}_{\delta}\right) \gamma \phi(A) .
\end{aligned}
$$


Without loss of generality we can choose $\delta$ so small that for any $N$ points $z_{i} \in M_{\delta}, 1 \leq i \leq N$, such that the balls $B\left(z_{i}, \delta\right)$ are disjoint, we can find $z_{N+1} \in M_{\delta}$ with

$$
B\left(z_{N+1}, \delta\right) \cap \bigcup_{1 \leq i \leq N} B\left(z_{i}, \delta\right)=\varnothing .
$$

For such a $\delta$ it is clear from (2) and the definition of $\mu_{\delta}$ that $P\left(\boldsymbol{x}, \mathcal{D}_{\delta}\right)>\mu_{\delta}$, which means that we can take $\gamma_{N+1}:=\mu_{\delta} \gamma>0$.

Now the assertion of part (ii) of the theorem is an immediate consequence of Lemmas 2 and 3 . Theorem 1 is proved.

\section{Local behaviour - a crude explanatory model}

Simulations of the $v$-process with $S(v)=v^{\alpha}$ show avalanche-scale clustering leading to the formation of a permanent tight cluster when $\alpha>1$ and $N$ is sufficiently large, and a weak variable clustering when $\alpha \leq 1$. It would be of interest to obtain some insight into the causes of this phase-change type of phenomenon. In this section we will present a simplistic model at a physicist's level of rigour, which explains why such a transition occurs at the threshold value $\alpha=1$.

Let $A \subset M$ be some small, connected 'test region' and $N_{A}(\boldsymbol{x}):=\operatorname{card}\left(\left\{i: x_{i} \in A\right\}\right)$. We consider the evolution of $N_{A}\left(\boldsymbol{X}_{n}\right)$. Let

$$
\mathcal{S}_{B}(\boldsymbol{x}):=\sum_{x_{i} \in B} S\left(\lambda\left(C_{x_{i}}^{\boldsymbol{x}}\right)\right), \quad B \subset M .
$$

Given that $\boldsymbol{X}_{n}=\boldsymbol{x}$, at the following step of the process the probability of a point being lost from $A$ is

$$
\frac{\mathcal{S}_{A}(\boldsymbol{x})}{\mathcal{S}_{M}(\boldsymbol{x})},
$$

while the probability of a fresh point entering $A$ is $\mu(A)$. As the total number $N$ of points in the process is typically much larger than $N_{A}\left(\boldsymbol{X}_{n}\right)$, it is natural to expect that, owing to the effect of a law of large numbers, the relative fluctuations in $\mathcal{S}_{M}\left(\boldsymbol{X}_{n}\right)$ will be small compared to those in $\mathcal{S}_{A}\left(\boldsymbol{X}_{n}\right)$ (this is borne out by the results of simulations). So let us assume, for simplicity, that $\mathcal{S}=\mathcal{S}_{M}\left(\boldsymbol{X}_{n}\right)$ is constant.

We wish to find an approximation to (15) as a function of $N_{A}=N_{A}\left(\boldsymbol{X}_{n}\right)$. Another simplifying assumption (also supported by simulations) is that the conditional (given $N_{A}$ ) distribution of cell volumes for cells whose generators lie in $A$ is the same, modulo scale, for different values of $N_{A}$. That is, for the conditional (on $N_{A}$ ) distribution function of the volume $V$ of a randomly chosen cell with generator in $A$ we have

$$
\operatorname{Pr}\left(V \leq v \mid N_{A}\right)=g\left(v / m_{A}\right), \quad v>0,
$$

for some $g$, where $m_{A}=\mathrm{E}\left(V \mid N_{A}\right)$. Furthermore, we can take

$$
m_{A}=N_{A}^{-1} \mathrm{E} \sum_{X_{n, i} \in A} \lambda\left(C_{X_{n, i}}^{\boldsymbol{X}_{n}} \mid N_{A}\right) \approx \beta \lambda(A) / N_{A},
$$

where $\beta$ is a quantity dependent only on the geometry of $A$ and the order of magnitude of $N_{A}$. This reflects the fact that the union of the cells with generators in $A$ overlaps $A$ itself. A rough 
calculation shows that $\beta=1+O\left(N_{A}^{-1}\right)$. When $A$ is a square or a circle and $N_{A}$ is not often less than 20, $\beta$ can be considered to be in the range $(1.0,1.5]$. Appealing to the law of large numbers, we could write

$$
\mathcal{S}_{A}\left(\boldsymbol{X}_{n}\right) \approx N_{A} \mathrm{E}\left(S\left(\lambda\left(C_{X_{n, I}}^{\boldsymbol{X}_{n}}\right)\right) \mid N_{A}\right)=N_{A} \int_{0}^{\lambda(M)} S(v) \mathrm{d} g\left(v / m_{A}\right),
$$

where $I$ stands for the index of a 'typical' cell with a generator $X_{n, I} \in A$. Now, since $S(v)=v^{\alpha}$, using (16) the integral above becomes

$$
\begin{aligned}
\int_{0}^{\lambda(M)} v^{\alpha} \mathrm{d} g\left(v / m_{A}\right) & =m_{A}^{\alpha} \int_{0}^{\lambda(M) / m_{A}} w^{\alpha} \mathrm{d} g(w) \\
& \approx N_{A}^{-\alpha}(\beta \lambda(M))^{\alpha} \int_{0}^{\infty} w^{\alpha} \mathrm{d} g(w),
\end{aligned}
$$

making the natural assumption that the last integral converges. Combining these approximations, we get the following estimate for the probability that a point is removed from $A$ in one step:

$$
\mathcal{S}_{A}\left(\boldsymbol{X}_{n}\right) \mathcal{S}^{-1} \approx N_{A}^{1-\alpha}(\beta \lambda(M))^{\alpha} \mathcal{S}^{-1} \int_{0}^{\infty} w^{\alpha} \mathrm{d} g(w)=K N_{A}^{1-\alpha},
$$

for some constant $K$. Thus, if $\Delta N_{A}$ denotes the change in $N_{A}$ in one step of the $v$-process, we have

$$
\mathrm{E}\left(\Delta N_{A} \mid N_{A}\right) \approx \mu(A)-K N_{A}^{1-\alpha} .
$$

As the right-hand side of (17) is an increasing function of $N_{A}$ when $\alpha>1$, in this case we have a positive feedback condition for the mean of the number $N_{A}$ of points in our test region $A$. This means that the process is bound to quickly leave the 'intermediate' range of states characterised by diffuse, roughly uniform spatial point distributions - an observation that is in agreement with the simulation data. Note also that, once the 'destabilising mechanisms' have transformed the point distribution to a single (or a few) tight cluster(s), the assumptions on which the crude local model was based are no longer valid.

On the other hand, when $\alpha \leq 1$, (17) expresses either neutral $(\alpha=1)$ or negative feedback. Hence we expect 'local stability' from the process behaviour: small clusters of points will form and disappear without any 'global' dramatic changes for the whole picture. In such cases, we can expect $N_{A}$ to take values close to $N \mu(A)$, so we can estimate $K$ using the equation

$$
K(N \mu(A))^{1-\alpha} \approx \mu(A),
$$

from which we conclude that

$$
K \approx \mu(A)^{\alpha} N^{\alpha-1}, \quad \alpha \leq 1 .
$$

This approximation is reasonably well supported by simulations.

\section{Acknowledgements}

Research supported by the ARC Centre of Excellence for Mathematics and Statistics of Complex Systems. The authors are grateful to G. Last for interesting and useful discussions of the model. The first author is also grateful to the Institute of Stochastics at the University of Karlsruhe for its hospitality while he was visiting the department and when those discussions first took place. 


\section{References}

[1] Asmussen, S. (1987). Applied Probability and Queues. John Wiley, Chichester.

[2] Baddeley, A. J. ANd van Lieshout, M. N. M. (1996). A nonparametric measure of spatial interaction in point patterns. Statist. Neerlandica 50, 344-361.

[3] Borovkov, K. A. And Odell, D. A. (2007). Simulation studies of some Voronoi point processes. Acta Appl. Math. 96, 87-97.

[4] Du, Q., FABER, V. AND GunZburger, M. (1999). Centroidal Voronoi tessellations: applications and algorithms. SIAM Rev. 41, 637-676

[5] Hasegawa, M. and Tanemura, M. (1976). On the patterns of space division by territories. Ann. Inst. Statist. Math. 28, 509-519.

[6] Hotelling, H. (1929). Stability in competition. Econom. J. 39, 41-57.

[7] Meyn, S. P. and Tweedie, R. L. (1993). Markov Chains and Stochastic Stability. Springer, New York.

[8] Nagel, K., Shubik, M., Paczuski, M. And BaK, P. (2000). Spatial competition and price formation. Physica A 287, 546-562.

[9] OKABE, A. AND Suzuki, A. (1987). Stability of spatial competition for a large number of firms on a bounded two-dimensional space. Environ. Planning A 19, 1067-1082.

[10] OKabe, A., Boots, B., Sugihara, K. And Chiu, S. N. (2000). Spatial Tessellations: Concepts and Applications of Voronoi Diagrams. John Wiley, Chichester.

[11] RiPley, B. D. (1977). Modelling spatial patterns. J. R. Statist. Soc. B 39, 172-212.

[12] Stoyan, D., Kendall, W. S. and Mecke, J. (1995). Stochastic Geometry and Its Applications. John Wiley, Chichester.

[13] Thönnes, E. And van Lieshout, M. N. M. (1995). A comparative study of the power of van Lieshout and Baddeley's $J$-function. Biom. J. 47, 721-734.

[14] Zuyev, S. and Tchoumatchenko, K. (2001). Aggregate and fractal tessellations. Prob. Theory Relat. Fields 121, 198-218. 